\title{
$1 \quad$ Cultured deep-sea PVC bacteria shed light on eukaryogenesis
}

2 Rikuan Zheng ${ }^{1,2,3,4}$, Chong Wang ${ }^{1,2,3,4}$, Tianhang Zhang ${ }^{5}$, Yingqi Tan ${ }^{1,2,3,4}$, Chaomin Sun ${ }^{1,2,3,4 *}$

$3 \quad{ }^{1} \mathrm{CAS}$ and Shandong Province Key Laboratory of Experimental Marine Biology \&

4 Center of Deep Sea Research, Institute of Oceanology, Chinese Academy of Sciences,

5 Qingdao, China.

$6 \quad{ }^{2}$ Laboratory for Marine Biology and Biotechnology, Pilot National Laboratory for

7 Marine Science and Technology, Qingdao, China.

$8{ }^{3}$ College of Earth Science, University of Chinese Academy of Sciences, Beijing,

9 China.

$10{ }^{4}$ Center of Ocean Mega-Science, Chinese Academy of Sciences, Qingdao, China.

$11{ }^{5}$ College of Life Sciences, Qingdao University, Qingdao, China

12

13

14

16

17

18

19

20

21

22

23

24

25

26

* Corresponding author

Chaomin Sun Tel.: +86532 82898857; fax: +8653282898857.

E-mail address: sunchaomin@qdio.ac.cn 6 17

Key words: PVC superphylum, eukaryogenesis, nucleus, nucleolus, mitosis

Running title: PVC-based eukaryogenesis

0




\section{Abstract}

28 Evolutionary relationship between prokaryotes and eukaryotes continues to fascinate

29 biologists. Accumulated studies suggest a eukaryogenesis model based on the PVC 30 (Planctomycetes-Verrucomicrobia-Chlamydiae) bacteria. However, a decisive 31 PVC-based eukaryogenesis scenario has not yet been reported. Here, we isolated PVC 32 bacteria, unique for dividing by budding and for possessing developed endomembrane 33 systems, from the deep sea. In cultured PVC bacterial strains, we detected typical 34 eukaryotic organelle-like structures including endoplasmic reticulum, Golgi apparatus, 35 vesicles, vacuoles and actin/tubulin-based microfilaments. Strikingly, we observed a 36 nucleolus-containing nucleus in a Verrucomicrobia strain, which divides by mitosis. 37 Transcriptomic results further demonstrated abundant presence of genes associated with eukaryotic cellular processes including membrane fusion. We propose that the 39 prominent capability of membrane fusion drives eukaryogenesis by enabling PVC bacteria to evolve eukaryotic cellular features. 


\section{Introduction}

53 Life on earth originated from two kinds of organisms: prokaryotes and eukaryotes ${ }^{1}$.

54 Although eukaryotes are generally considered to have evolved from prokaryotes, how this occurred remains one of the greatest enigmas in biology $\mathrm{y}^{2-4}$. At the time of eukaryogenesis, the last eukaryotic common ancestor (LECA) had acquired a nucleus enclosed by a double membrane, membranous intra-cellular structures, mitochondria, and actin/tubulin-based cytoskeletal structures ${ }^{2}$. Moreover, the LECA also underwent intricate biological processes such as apoptosis, intron splicing, and meiosis ${ }^{2}$. Two competing hypotheses seek to explain the origin of eukaryogenesis: the endosymbiotic and the autogenic ${ }^{5,6}$. According to the autogenesis theory, the LECA evolved from a prokaryote through a gradual increase in cellular complexity that slowly accumulated over time $e^{1,2,7}$, and development of an endomembrane system that drove the compartmentalization of the nucleus ${ }^{6}$. Conversely, the endosymbiotic hypotheses propose that a moderately large and anaerobic prokaryote engulfed aerobic bacteria, some of which escaped digestion and instead became stabilized as endosymbionts, eventually evolving into mitochondria ${ }^{8,9}$. Testing these theories has been difficult given the reliance of canonical models unlikely to capture the wider spectrum of prokaryote cell biology and the paucity of known intermediate stages in the prokaryote-to-eukaryote transition ${ }^{1,10}$. However, if eukaryotes evolved from

71 prokaryotes, then there must have been viable organisms with intermediate cellular structures and life styles between prokaryotes and eukaryotes ${ }^{1,10}$, and investigating such intermediates therefore holds the potential to decode the mysterious eukaryogenesis process.

Members within the PVC superphylum (which also includes bacteria from Lentisphaerae and Kirimatiellaeota as well as some uncultured candidate phyla) are examples of bacteria with features usually associated with eukaryotes or archaea, or both $^{10-12}$, and hence represent exceptions to how we define bacteria. Consistently, earlier work proposed that the ancestor of the PVC bacteria diverged by developing distinct features that would later become known as archaeal, eukaryotic, or shared 
81 between them ${ }^{10}$. The PVC bacteria are thought to be the sister taxa to the LAECA

82 lineage and the root of the Archaea and eukaryotes ${ }^{10}$. However, the objectivity and 83 accuracy of this scenario need to be further clarified through culturing PVC bacteria 84 that possessing more significant eukaryotic signatures (e.g. nucleus and other complex membranous cellular organelles).

Given that the deep sea is one of the most likely environments to harbor intermediates between prokaryotes and eukaryotes ${ }^{1}$ and has been proposed to be the proper place of life origin and stepwise evolution ${ }^{13}$, we here set out to culture deep-sea PVC bacterial strains. We found that these presented with many typical eukaryotic organelle-like structures, and specifically, detected a nucleolus-containing nucleus in a Verrucomicrobia strain, which divides through a mitosis-like manner with the involvement of the nucleolus. Lastly, we discussed the possible pathways of PVC-based eukaryogenesis driven by cell fusion.

\section{Results and Discussion}

Abundant eukaryotic signatures observed in deep-sea PVC bacteria. To closely assess presence of eukaryotic cellular features, we cultured four PVC bacteria, namely two Planctomycetes strains, one Verrucomicrobia strain and one Lentisphaerae strain isolated from deep-sea cold seeps (Fig. S1A). Through TEM analyses, we concluded that Planctomycetes and Verrucomicrobia strains likely divide by budding (Fig. S1B) and that membrane structures in PVC bacteria are both more complex and extended than those of typical Gram-positive (Expanded Data Fig. S1C, panel 1) or -negative bacterium (Expanded Data Fig. S1C, panel 2). PVC bacteria also have a condensed and intact nucleoid-like structure (Expanded Data Fig. S1C, panels 3-5), as exemplified by a typical nucleus-like organelle containing a well-shaped nucleolus in the Verrucomicrobia strain ZRK36 (Expanded Data Fig. S1C, panel 6), similar to those in fungal and human cells (Expanded Data Fig. S1C, panels 7-8). Additionally, eukaryotic signatures including endoplasmic reticula (ER) like-, vesicle like-, or vacuole like-organelles are present in Planctomycetes strain ZRK32 (Expanded Data Fig. S1C, panel 4). Although the presence of the nucleus and other membrane-bound organelles 
110 are hallmark features of eukaryotic cells, a recent cross-species proteomic analysis of

111 the prokaryote nuclear matrix structure identified a significant fraction of such proteins

112 evolutionary related to counterparts in eukaryotes ${ }^{14}$. In line with emerging studies

113 highlighting unique properties of PCV bacteria $^{12}$, we conclude that PVC strains are

114 differentiated from how bacteria are conventionally defined by their abundant

115 eukaryotic signatures as well as a high structural complexity. Intrigued by the distinct

116 cell biology we had detected in Planctomycetes strain ZRK32 and Verrucomicrobia

117 strain ZRK36, we decided to focus on these two strains for further studies.

118 Eukaryotic signatures of Plancomycetes. To explore its additional eukaryotic 119 features, we cultured Planctomycetes strain ZRK32 in both basal and rich media and 120 analyzed the cellar ultra-structures in ultrathin TEM sections. We clearly detected 121 several eukaryotic membrane-trafficking machinery components including the Golgi

122 apparatus and the ER (Fig. 1A and Expanded Data Figs. S2-S3). In the 123 prokaryote-to-eukaryote evolutionary transition, the emergence of an endomembrane 124 system resembling the Golgi and ER was a pivotal event ${ }^{9}$, as internal tubular networks 125 enable specialization and compartmentalization of biochemical processes, as well as a 126 varied surface matrix supporting rich intracellular processes and signaling cascades ${ }^{15,16}$ 127 In eukaryotic cells, highly specialized vesicle trafficking protein complexes direct 128 transport between the ER and Golgi ${ }^{2}$. In the strain ZRK32, we identified both a vesicle 129 like-structure (Fig. 1A and Expanded Data Figs. S4A-C) and a vacuole-like structure 130 (Fig. 1A and Expanded Data Figs. S4D-F). Vacuoles are highly dynamic and 131 pleiomorphic ${ }^{17}$, with a size that varies depending on the cell type and growth conditions 132 (Expanded Data Figs. S4D-F). Vacuoles store cellular components such as proteins, 133 sugars etc., and play essential roles in plants response to different biotic/abiotic 134 signaling pathways ${ }^{17}$. The presence of vacuoles in strain ZRK32 suggests that 135 Planctomycetes bacteria might have adopted a eukaryotic mechanism for nutrient 136 metabolism and signal transduction. In the TEM sections, we also observed several 137 types of actin/tubulin-derived fibrous structures (Fig. 1A, panels 7 and 8), suggesting 138 that Planctomycetes members also evolved sophisticated cytoskeletal machineries, 
bioRxiv preprint doi: https://doi.org/10.1101/2021.11.19.469327; this version posted November 19, 2021. The copyright holder for this preprint (which was not certified by peer review) is the author/funder. All rights reserved. No reuse allowed without permission. strains, and proposed to be involved in specific locomotion and engulfment processes $^{18}$.
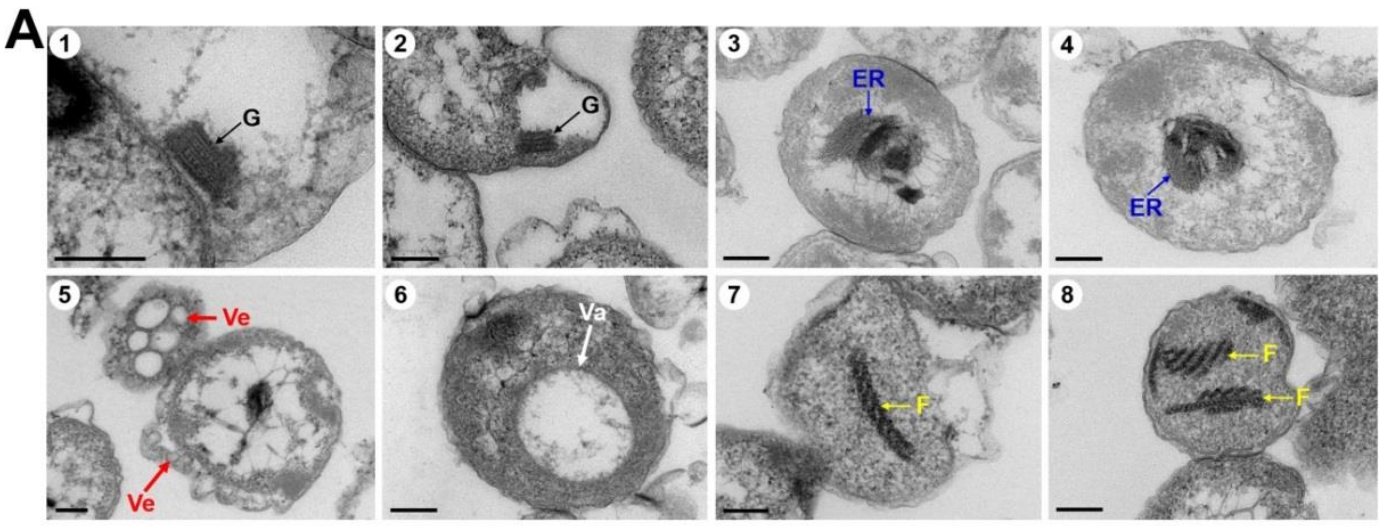

B
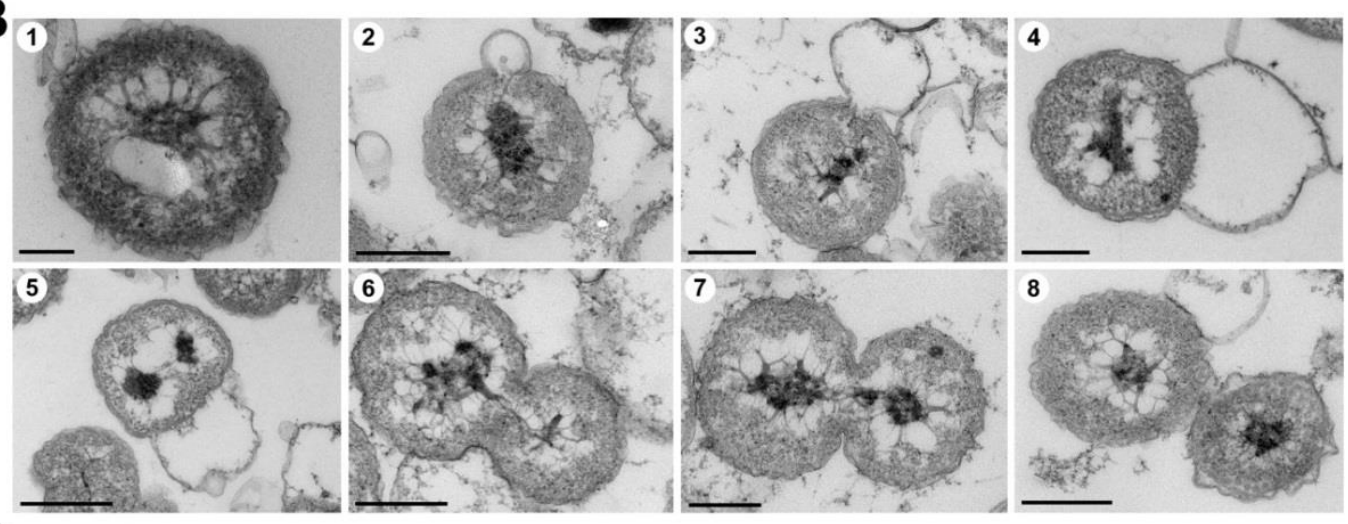

C
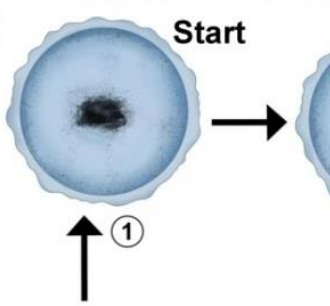

(2)
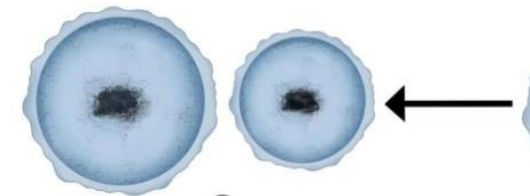

(8)
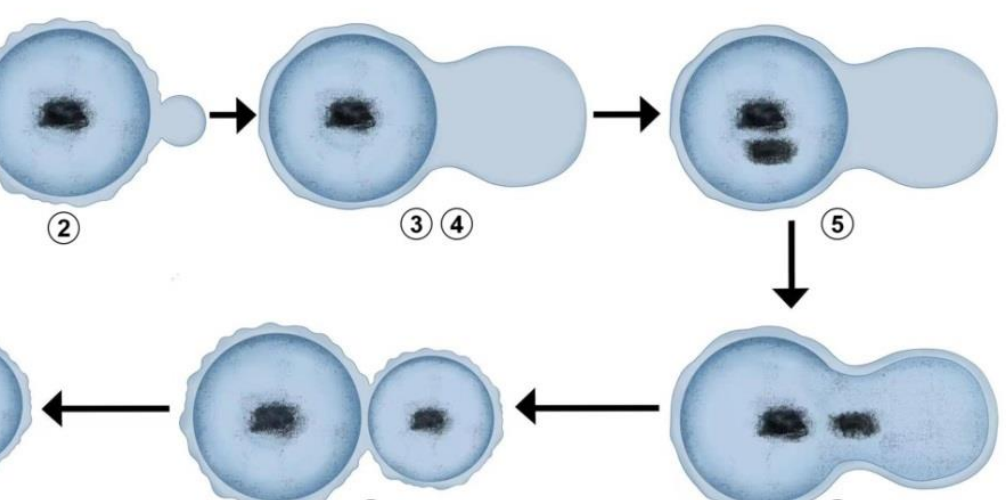

(7)

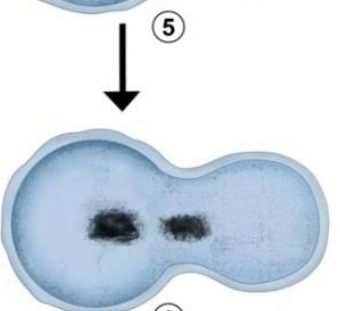

(6)

142 Fig. 1. The unique eukaryotic signature and cell division pattern of Planctomycetes strain ZRK32. (A) Ultrathin TEM sections showing Golgi apparatus like- (indicated with $\mathrm{G}$ in panels 1 and 2), endoplasmic reticula like- (indicated with ER in panels 3 and 4), vesicle like- (indicated with Ve in panel 5), vacuole like- (indicated with Va in panel 6), and microfilaments-like (indicated with F in panels 7 and 8) organelles observed in the cell of Planctomycetes strain ZRK32. (B) Ultrathin TEM sections showing the process of particular polar budding division (panels 1-8) conducted by strain ZRK32. Representative pictures representing different phases of cell division are shown. Scale bars are $200 \mathrm{~nm}$ in panels A and B. (C) The proposed model of cell division of

150 Planctomycetes strain ZRK32 based on the TEM observation shown in panel $\mathrm{B}$. The numbers are the same to those shown in panel B. 
The Planctomycetes strain ZRK32 is shown to divide by budding as previously reported ${ }^{19}$. In the beginning of division, the membrane extends and forms a bulge 154 region, which becomes larger until its size is similar to that of the mother cell (Figs. 1B and $1 \mathrm{C}$, panels 1 to 4 ). Meanwhile, the genetic materials within the nucleoid are

156 duplicated and equally divided between the mother and daughter cells along with other cytoplasmic contents (Figs. 1B and 1C, panels 5 to 8), after which the daughter cell completely separates from the mother cell to finalize a cell division cycle. Here, we asked whether genes associated with eukaryotic features and cell division are functional during growth. To this end, we performed a comparative transcriptomic analysis of strain ZRK32 cultured in basal and rich media (Fig. 2A). Overall, the transcriptomic profiles revealed significant regulation of genes encoding ER associated

163 proteins, Golgi vesicle transport, vacuole sorting, SNARE-associated Golgi protein, 164 cadherin domain, and Rab/Rho GTPases (Fig. 2B). While SNARE-associated proteins 165 play specific roles in vesicle fusion ${ }^{20}$, the small Ras GTPase superfamily, one of the 166 largest protein families in eukaryotes, is broadly involved in various regulatory processes, including cytoskeleton remodelling, signal transduction, nucleocytoplasmic transport and vesicular trafficking ${ }^{21}$. Additionally, genes associated with actin/tubulin are strongly upregulated (Fig. 2C). In eukaryotes, both actin and tubulin polymerize to form filaments that plays central roles in cellular motility, cytoskeleton formation and 171 phagocytosis $^{22}$. Their presence and upregulation in strain ZRK32 indicate that filament 172 structures might play a critical role in determining its cell shape and movement. In 173 microbial cell division, members of the tubulin-homologue Fts protein family assemble 174 at the future site of cell division to form a contractile ring, known as the $\mathrm{Z}$ ring ${ }^{23}$. 175 Consistent with earlier work ${ }^{19}$, we did not detect the FtsZ isoform, but numerous other 176 Fts- proteins were present and upregulated in rich medium-supported growth (Fig. 2D).

177 Within the group of proteins associated with cytoskeletal function, the gene encoding 178 for VgrG1, an actin cross-linking protein known for a role in the bacterial type VI 179 secretion system, to be the most upregulated ${ }^{24}$. We also found that the bacterial 180 actin-analogue MreB, was upregulated in strain ZRK32 (Fig. 2C). Peptidoglycan 
182 filaments such as MreB that participate in its formation and degradation ${ }^{25}$. MreB is 183 probably not generally required for either cell division or cell-shape determination in 184 Planctomycetes $^{19}$, but its function in cellular processes is diversified in different Planctomycetes strains.
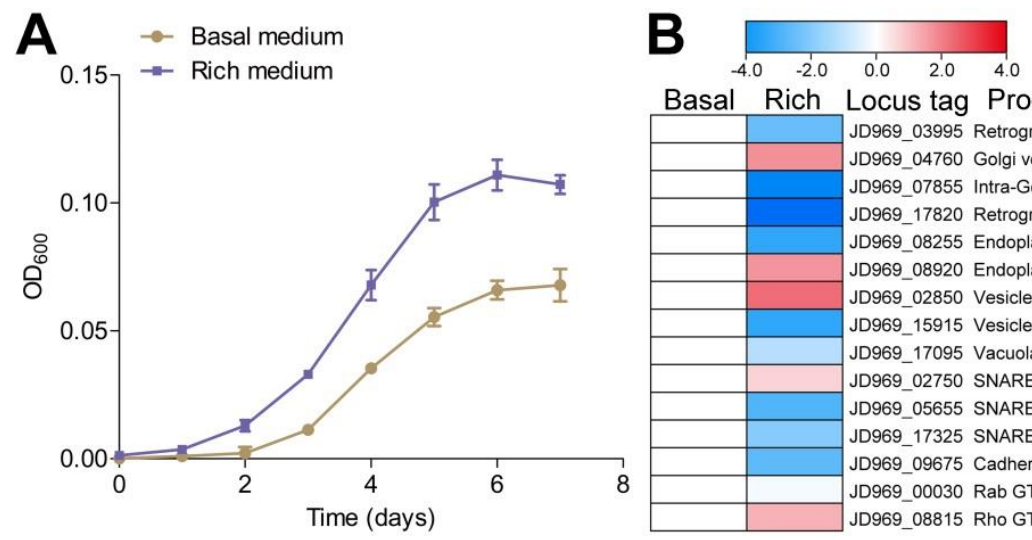

\section{C}

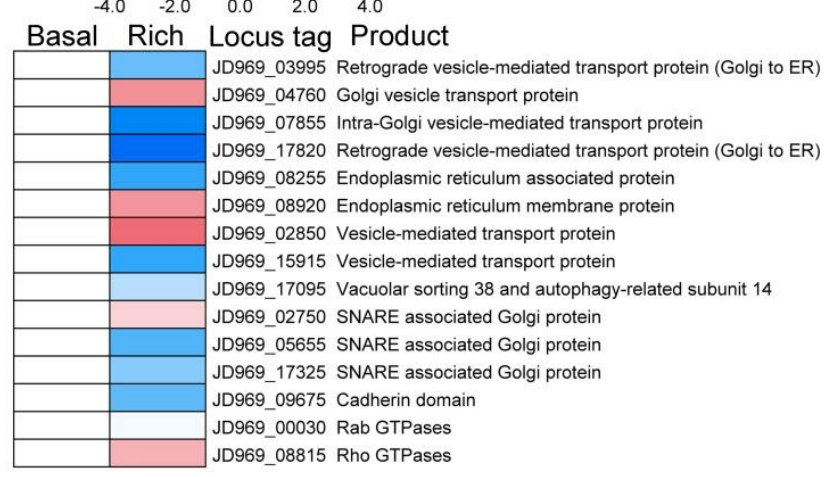

\begin{tabular}{|l|l|l|l}
\hline & JD969_02150 Tubulin complex assembly protein \\
\hline & JD969_05915 Tubulin binding protein \\
& JD969_10415 Tubulin-tyrosine ligase family protein \\
& JD969_20355 Tubulin complex assembly protein \\
& JD969_05660 Actin cross-linking toxin VgrG1 \\
& JD969_07810 Actin binding protein \\
\hline & JD969_08220 Actin binding protein \\
\hline & JD969_12085 Vulnibactin protein \\
\hline & JD969_12270 Actin binding protein \\
\hline & JD969_15705 Arp2/3 complex-mediated actin nucleation \\
\hline & JD969_18040 Actin binding protein \\
\hline & JD969_00595 MreB protein \\
\hline & JD969_06685 MreB protein \\
\hline & JD969_16405 MreB protein \\
\hline & JD969_17290 Rod shape-determining protein MreB \\
\hline & JD969_18535 MreB protein
\end{tabular}

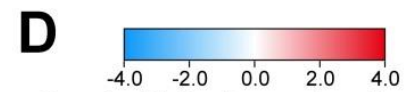

\begin{tabular}{|l|l|l}
\multicolumn{1}{c}{ Basal Rich Locus tag Product } \\
\hline & & JD969_15485 Cell division protein FtsH \\
& JD969_06075 Cell division protein FtsJ \\
& & JD969_00285 Cell division protein FtsK \\
& JD969_01015 Cell division protein FtsK \\
\hline & & JD969_01490 Cell division protein FtsK \\
\hline & & JD969_05860 Cell division protein FtsK \\
\hline & JD969_07060 Cell division protein FtsK \\
\hline & JD969_08245 Cell division protein FtsK \\
\hline & JD969_14170 Cell division protein FtsK \\
\hline & JD969_15230 Cell division protein FtsK \\
\hline & JD969_11500 Cell division protein FtsL \\
\hline & JD969_12120 Cell division protein FtsP \\
\hline & JD969_14360 Cell division protein FtsW \\
\hline & JD969_14075 Cell division protein FtsX \\
\hline & JD969_05640 Cell division protein FtsY
\end{tabular}

Fig. 2. Transcription assays of genes associated with eukaryotic signatures and cell division of Planctomycetes strain ZRK32. (A) Growth assays of strain ZRK32 cultivated in either rich medium or basal medium. (B) Transcriptomics based heat map showing differentially expressed genes encoding proteins associated with eukaryotic signatures in strain ZRK32. (C)

191 Transcriptomics based heat map showing differentially expressed genes encoding proteins associated with cytoskeleton in strain ZRK32. (D) Transcriptomics based heat map showing differentially expressed genes encoding key proteins associated with cell division in strain ZRK32.

194 Eukaryotic signatures of Verrucomicrobia. Similar to what we observed in

195 Planctomycetes strain ZRK32, ultrathin TEM sections of Verrucomicrobia strain

196 ZRK36 cells revealed presence of ER like- and vacuole like-structures (Fig. 3A).

197 However, the most striking feature of Verrucomicrobia strain ZRK36 is its well-shaped 
nucleus containing a nucleolus-like structure (Fig. 3A, panels 1-2). Although the nucleoid of Planctomycetes is considered to be more like eukaryotic nuclei ${ }^{26}$, the kind of clear and intact nucleus structure that we observed in strain ZRK36 has to our knowledge not been reported in any other bacteria. The nucleus, which contains almost all of the cells' genetic material, is a remarkably complex structure, and a hallmark feature of eukaryote cells ${ }^{26,27}$. Strikingly, we also observed a structure resembling the nucleolus (Fig. 3A, panels 1-3), whose main function in eukaryote cells is ribosome biogenesis and ribosomal RNA (rRNA) synthesis ${ }^{28}$. In the initial stage of budding division, the nucleolus in strain ZRK36 is duplicated and the new copy along with cytoplasmic materials enters into the newly formed budding structure (Fig. 3B, panel 2). Thereafter, the size of budding becomes almost equal to that of the mother cell (Fig. 3B, panel 3). At that stage, the genetic materials inside the nucleolus are disassembled, thereby directing development of the daughter cell (Fig. 3B, panels 4 and 5). Thereafter, the formation of a new nucleus is initiated, as demarcated by a condensed nucleolus (Fig. 3B, panel 6). Subsequently, the spindle fibers between the old and new nuclei are extended along with separation of the two nuclei (Fig. 3B, panel 7). Finally, the extended spindle fibers disappear and the daughter cell becomes thoroughly segregated from the mother cell, completing the division process (Fig. 3B, panels 8-10). At the end of mitosis, eukaryotic cells reshape the intact nucleus by segregating the copies of the replicated genome into two new nuclear compartments, which splits into two in a "closed mitosis"29. Here, we found that the ZRK36 strain undergoes a meiosis-like cell division process that is highly similar to that of budding yeast (Figs. 3C and 3D), supporting the conclusion that this PVC bacterium evolved to adapt a eukaryotic cell division mode. Meiosis is widely considered to be a core process unique to eukaryotes, and consequently expected to be absent from all known prokaryote ${ }^{2}$. However, on the basis of our high-resolution morphology analysis of strain ZRK36 in ultrathin TEM sections, we conclude that some PVC bacteria throughout evolution acquired eukaryotic organelle-like structures as well as cell cycle progression and cell division modes believed to be solely conducted in eukaryotes. 


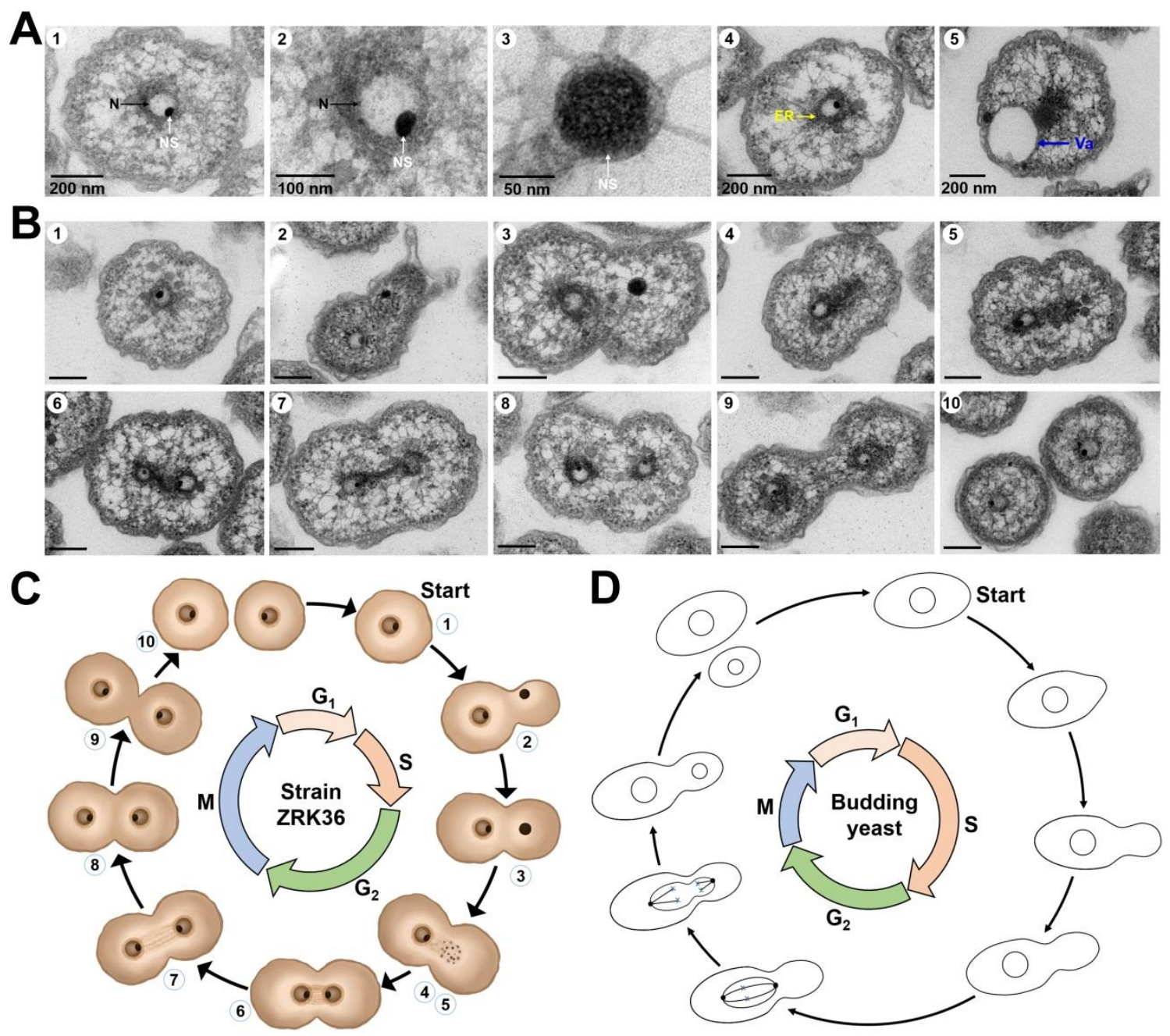

Fig. 3. The unique eukaryotic signatures and cell division pattern of Verrucomicrobia strain ZRK36. (A) Ultrathin TEM sections showing, nucleus like- (indicated with $\mathrm{N}$ in panels 1 and 2), nucleolus like- (indicated with NS in panels 1 to 3), endoplasmic reticula like- (indicated with ER in panel 4) and vacuole like- (indicated with Va in panel 5) organelles observed in the cell of Verrucomicrobia strain ZRK36. (B) Ultrathin TEM sections showing the process of a typical mitosis manner (panels 1-10) conducted by Verrucomicrobia strain ZRK36. Representative pictures representing different phases of cell division are shown. Scale bars are $200 \mathrm{~nm}$. (C) The proposed model of cell division conducted by Verrucomicrobia strain ZRK36 based on the TEM observation shown in panel B. The numbers are the same to those shown in panel B. (D) Typical cell division process conducted by the budding yeast. G1, S, G2 and M in panels $\mathrm{C}$ and D represent different phases of meiosis.

It's noteworthy that many genes that encode factors associated with eukaryotic organelles and unique cell biology are significantly regulated during growth (Fig. 4B), strongly suggesting that they function similarly in processes that contribute to the growth of strain ZRK36 (Fig. 4A). Among growth-regulated genes, NOG1, which encodes the nucleolar GTPase Nog1, a coordinator factor in ribosome formation, is 
243 known to be present in the nucleolus in humans and yeast ${ }^{30,31}$. In yeast, the NOG1 gene 244 is essential for cell viability and plays an important role in nucleolar functions ${ }^{30}$. Based 245 on its described function in eukaryotic cells, we propose that the NOG1 homologue in 246 strain ZRK36 plays a similar nucleolus-directed role in cell division. Similarly, we 247 found that the gene encoding centromere protein $\mathrm{H}(\mathrm{CENPH})$ is also regulated, 248 implicating mitosis as another distinct eukaryotic process in strain ZRK36 (Fig. 4B). 249 CENPH is as a component of the active centromere complex, which directs kinetochore 250 assembly on the centromeric chromatin to ensure faithful chromosome segregation 251 during mitosis ${ }^{32,33}$. Given the combined electron microscopy analysis and 252 transcriptomic profiling, we propose that strain ZRK36 undergoes a eukaryotic-like 253 mitosis process.
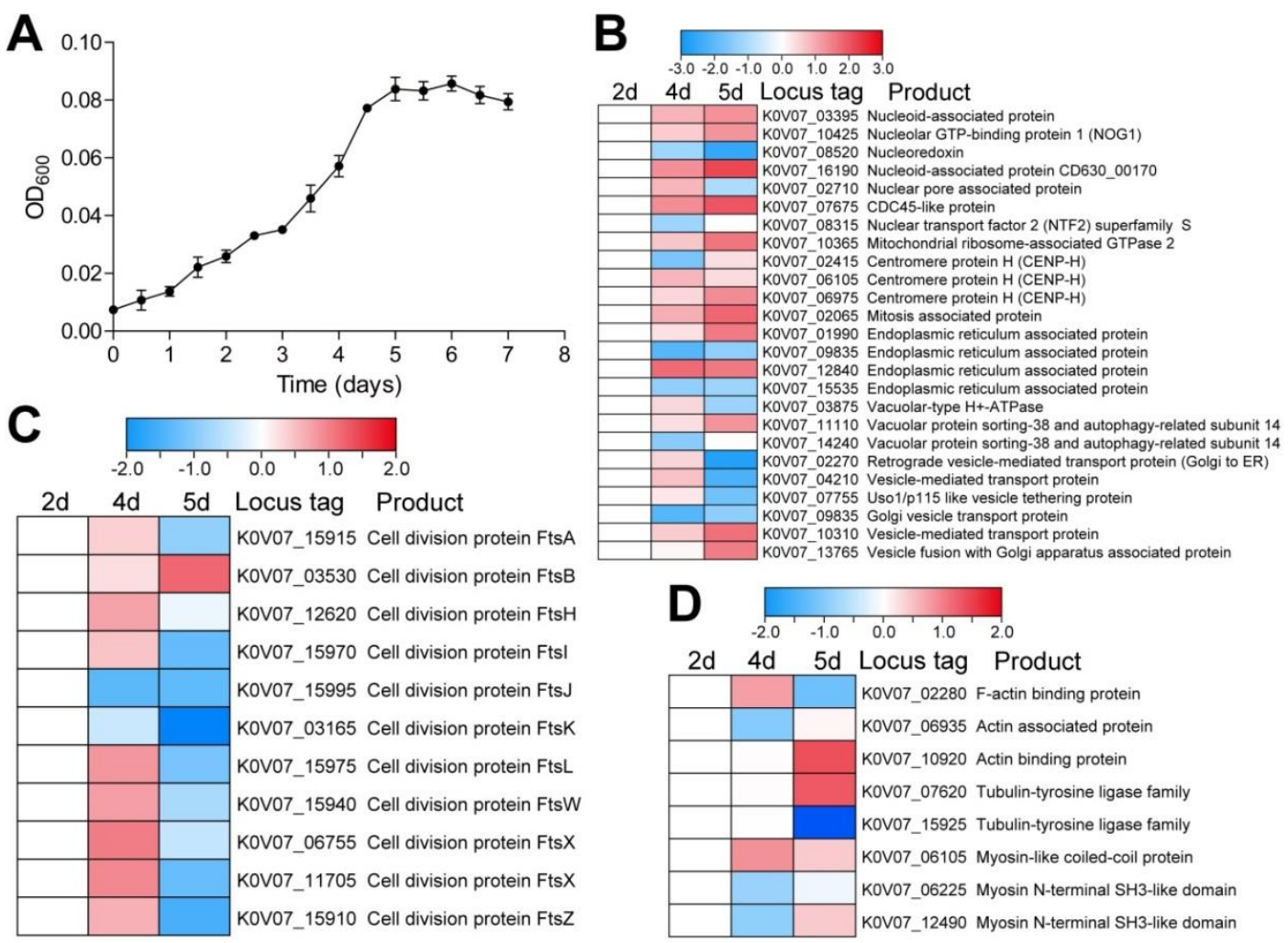

Fig. 4. Transcription assays of genes associated with eukaryotic signatures and cell division of Verrucomicrobia strain ZRK36. (A) Growth assays of strain ZRK36 cultivated in basal medium. (B) Transcriptomics based heat map showing differentially expressed genes encoding proteins associated with eukaryotic signatures in strain ZRK36. (C) Transcriptomics based heat map showing differentially expressed genes encoding key proteins associated with cell division in strain ZRK32. (D) Transcriptomics based heat map showing differentially expressed genes encoding proteins associated with cytoskeleton in strain ZRK36. 
263 ZRK36 where it likely participates in the process of cell division together with other 264 Fts-proteins (Fig. 4C) and actin/tubulin associated proteins (Fig. 4D). Since FtsZ is a 265 tubulin family protein, it is conceivable that early gene duplication giving rise to both 266 tubulins and FtsZ occurred in some Verrucomicrobia bacteria. Taken together, our 267 observations of abundant eukaryotic features in strains ZRK32 and ZRK36 illustrate how deep-sea PVC bacteria challenge the knowledge we have gained by studying conventional model organisms and suggest that these provide good models to explore eukaryogenesis.

271 Significant membrane fusion capability of PVC bacteria. A feature characteristic of 272 PVC bacteria, but not typically seen in other bacteria, is their endomembrane system, 273 which is particularly well developed in some species ${ }^{12,23,34}$. Unlike in prokaryotes,

274 fusion of membranous organelles is the basis of life in eukaryotes, with numerous processes hinging on the flawless timing and operation of membrane fusion ${ }^{35}$. Notably, 276 all deep-sea PVC members cultured in this study have well-developed endomembrane system and show diverse abilities of membrane fusion. In the Lentisphaerae strain zth2,

278 we captured several extracellular protrusions that mediate cell-cell interactions and 279 membrane fusion (Fig. 5A), which enables the formation of an integrated channel for 280 exchanging cellular material (Fig. 5A). Indeed, in the inside-out theory of eukaryote 281 evolution, protrusions that enable material exchange are suggested to function as a 282 cellular mechanism that engulfs mitochondria-like bacteria ${ }^{6}$. Here, we find that 283 Planctomycetes strain ZRK32 has a remarkable endocytosis capability, enabling 284 vesicle formation from the extracellular environments via cell fusion and forming a 285 larger size vesicle (Fig. 5B). We speculate that superiority in capturing extracellular 286 nutrient and phagocytose smaller cells might endow endomembrane system-carrying 287 bacteria with both an evolutionary and survival advantage. In Verrucomicrobia strain 288 ZRK36, we detect a dramatic cell fusion capability, allowing the formation of giant 289 cells with several-fold size of the original cell (Expanded Data Fig. 5C and Fig. S5). In 290 addition, several nuclei-like organelles are capable of fusing into a bigger size nucleus 
291 (Fig. 5C, panel 6). Moreover, giant cells are more prevalent in the stationary phase than

292 in the logarithmic phase.
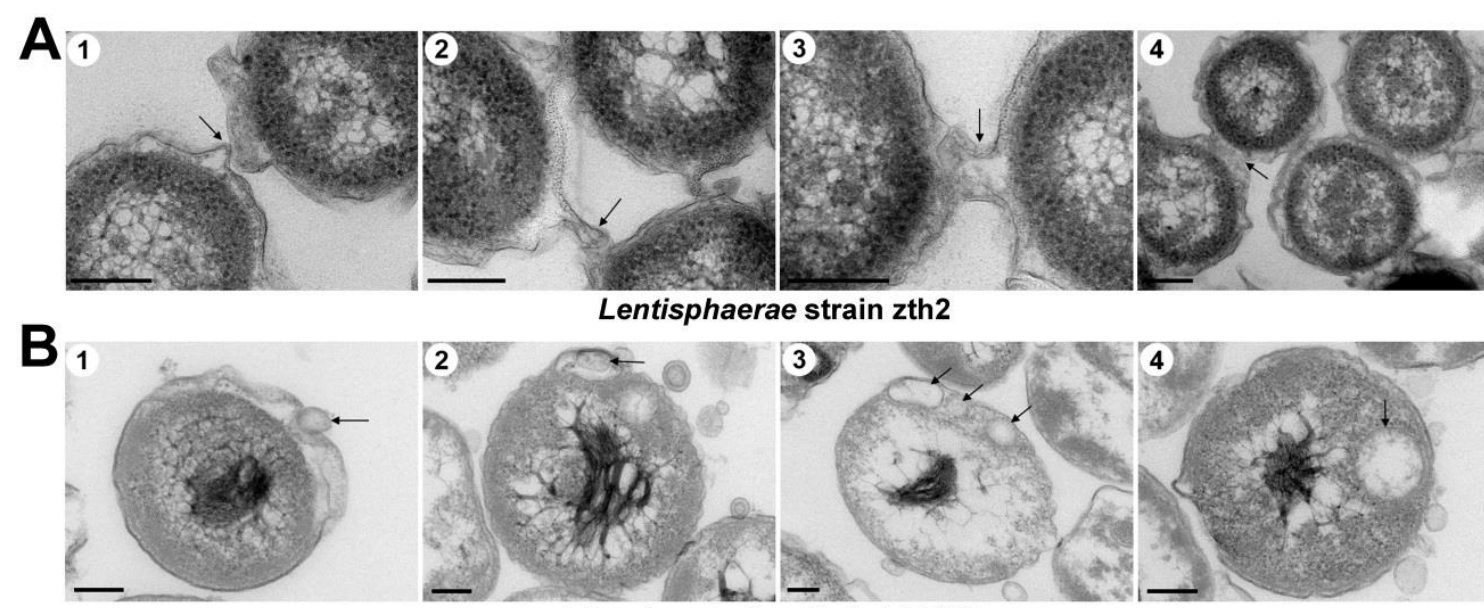

Planctomycetes strain ZRK32

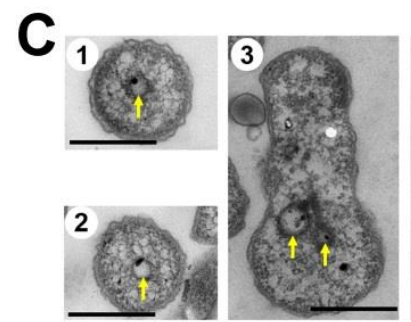

D
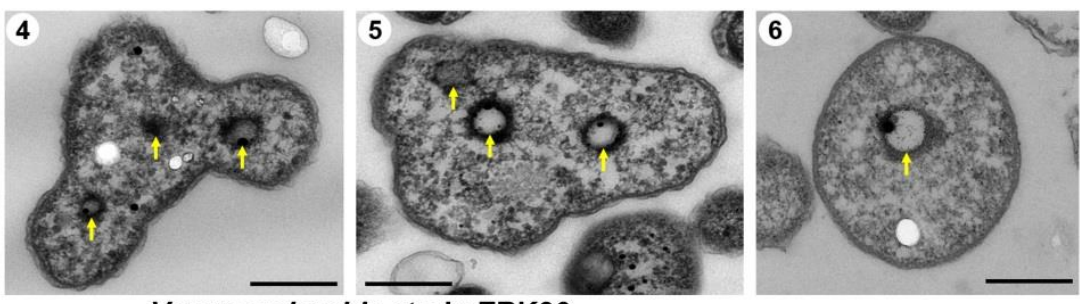

Verrucomicrobia strain ZRK36
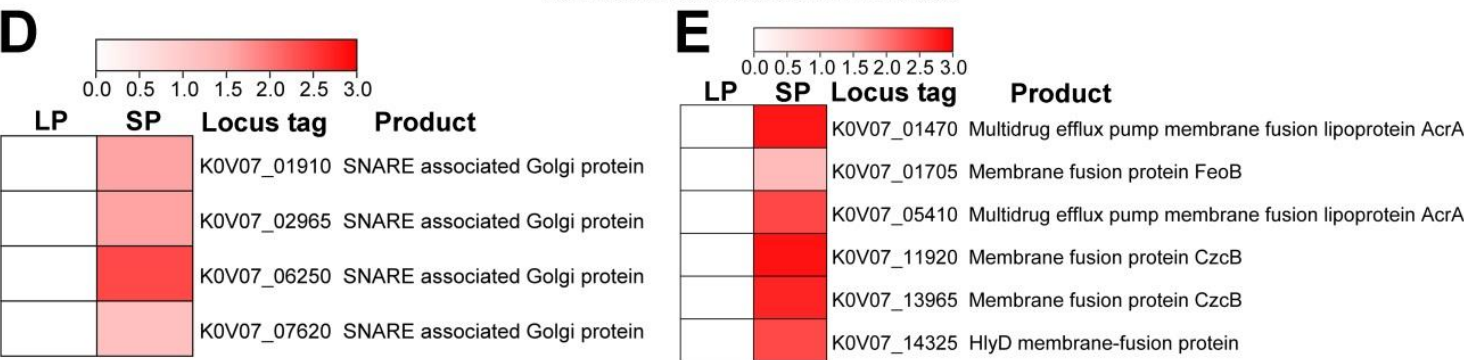

Verrucomicrobia strain ZRK36

294 Fig. 5. Diverse membrane fusion manners conducted by PVC bacteria. (A) Ultrathin TEM sections showing the process of membrane fusion directed by the protrusions around the cell of Lentisphaerae strain zth2. The arrows in panels 1 to 4 indicate the position of membrane fusion. (B) Ultrathin TEM sections showing the endocytic process through a membrane fusion manner conducted by Planctomycetes strain ZRK32. The arrows in panels 1 to 4 indicate the positions of vesicle. (C) Ultrathin TEM sections showing the inter-species phagocytosis conducted by Verrucomicrobia strain ZRK36. The arrows in panels 1 to 6 indicate the positions of nucleus like-organelles. (D) Transcriptomics based heat map showing differentially expressed genes encoding SNARE-associated Golgi proteins in Verrucomicrobia strain ZRK36. Transcriptomics based heat map showing differentially expressed genes encoding proteins associated with membrane fusion in Verrucomicrobia strain ZRK36. "LP" indicates the logarithmic phase; "SP" indicates the stationary phase.

Consistent with our phenotypic analysis (Fig. 5C), the transcriptome profiles reveal that membrane fusion genes are significantly upregulated (Figs. 5D and 5E). 
308 Among these, four genes encoding SNARE-associated proteins are prominently 309 up-regulated in the stationary phase relative to the logarithmic phase (Fig. 5D). 310 Membrane fusion, a universal feature of eukaryotic protein trafficking, is mediated by 311 SNARE-associated proteins that embed in opposing membranes to spontaneously drive 312 membrane fusion and cargo exchange ${ }^{36}$. Additionally, the expressions of other 313 membrane fusion proteins/lipoproteins were also up-regulated in the stationary phase 314 (Fig. 5E). Based on these analyses, we conclude that Verrucomicrobia strain ZRK36 315 has evolved a significant cell fusion capability, enabling it to form more complicated 316 and larger cells, and executing processes resembling phagocytosis, an eukaryotic 317 innovation conserved from unicellular protists to animals, that enabled eukaryotes to 318 feed on other organisms ${ }^{18}$. Presumably, other PVC bacteria might also evolve similar 319 membrane fusion strategies to increase cell complex and size, and in turn facilitating 320 eukaryogenesis.

321 PVC-based eukaryogenesis. PVC bacteria have long been deemed close associates of 322 the eukaryote ancestor ${ }^{12,23,26}$. Although eukaryogenesis-predictive features are 323 common in PVC bacteria, their implications and real functions have not been 324 adequately addressed. Based on our data and recent related work, we speculate that 325 eukaryogenesis could have evolved in PVC bacteria through two core mechanisms: 326 inter-species and cross-species fusions. In inter-species fusion, two PVC bacteria, each 327 with a well-developed nucleus (e.g. Verrucomicrobia strain ZRK36) first fuses to form 328 a larger cell, which then sequentially fuses with bacteria that has acquired either 329 eukaryote-like endomembrane systems or other organelles to form a complex 330 eukaryotic cell (Fig. 6A). In cross-species fusion, a PVC bacterium with a developed 331 nucleus first fuses with a PVC or a related strain to form a larger cell with developed 332 eukaryotic features, which then sequentially fuses with bacteria that has acquired a 333 nucleus-like or other organelle-like structures to form a complex eukaryotic cell (Fig. 334 6B). In the hypothetical LECA, the endomembrane systems, including the ER, Golgi 335 complex and the main components of the endocytosis and recycling systems, were 336 already present ${ }^{2}$, but the evolutionary events preceding this transition are a matter of 
debate. Based on comparative genomic analysis, earlier work proposed the Asgard superphylum, belonging to archaea (a separate domain from bacteria and eukaryotes that lack nuclei), as a candidate host cell that merged with a bacterial mitochondrial endosymbiont to generate the first eukaryotic cell ${ }^{37}$. This scenario supports a 2D model of evolution in which bacteria and archaea evolved along two separate branches of the tree of life, and eukaryotes evolved from archaea ${ }^{37}$. Here, at a cell biological and transcriptome level, we discovered more developed eukaryotic features in deep-sea PVC bacteria than previously reported, most notably a nucleolus-containing nucleus and mitosis, supporting the alternative and recent hypothesis of PVC-based eukaryogenesis ${ }^{10}$. Based on our data, we propose a modified PVC-based linear (1D) scenario of eukaryogenesis (Fig. 6C). In our model, we propose that the last universal common ancestor (LUCA) diverged into two linages: bacteria with very few archaeal and eukaryotic features, and the last PVC common ancestor (LPCA), with abundant archaeal or eukaryotic features, or both. At the subsequent evolutionary branch point, the LPCA diverged into the last modern PVC common ancestor (LMPCA) that had lost most eukaryotic signatures, and the last archaeal and eukaryotic common ancestor (LAECA). In this scenario, PVC bacteria are related to intermediates in both LPCA derived lineages: LMPCA and LAECA, the former less complex, and the latter more complex, progressively accumulating eukaryotic or archaeal features and thereby increasing its cellular complexity. However, intermediates diverging towards LMPCA or LEACA likely share many common features initially, resulting in universal communication between these two groups through cell fusions (as indicated with dotted lines between the LMPCA and LEACA branches). Indeed, findings in recent years revealed that eukaryotic ancestors more easily exchanged genes when they were more closely related, particularly at the start of their diversification ${ }^{2}$. Throughout PVC-based eukaryogenesis, ubiquitous and multilateral fusions would be expected to create chimeric cells containing increasingly complicated genetic materials and other organelle-like structures. We propose that the PVC members cultured in the present study (Planctomycetes strain ZRK32 and Verrucomicrobia strain ZRK36) might represent intermediate states between the LPCA and the LAECA as the LPCA became 
367 more "eukaryotic" with a gain of complexity (Fig. 6C) ${ }^{10}$. The LPCA as well as 368 intermediates between the LPCA and the LAECA will unlikely have evolved a 369 sophisticated nucleus or eukaryote-associated features ${ }^{38}$; instead these stages are likely 370 evolutionary "works in progress" until the very end of eukaryogenesis, which remains 371 as the main gap in the history of life on the Earth (Fig. 6C). Hence, we propose that 372 future exploration of prokaryotic diversity should focus on the later time line between 373 the LPCA and the LAECA, which diverged to the last archaeal common ancestor 374 (LACA) and the last eukaryotic common ancestor (LECA). In the PVC-based linear 375 tree of life, archaea have lost most of the eukaryotic features, with Asgard having lost 376 the least and diverged less ${ }^{10}$, consistent with their sheer number of eukaryotic features 377 and the phylogenetic proximity of these eukaryotic features to eukaryotes (Fig. 6C) ${ }^{37}$. 378 The PVC-based 1D scenario we outlined here does not deny the proximity of Asgard to 379 Eukarya, nor does it question the notion that eukaryotes may have evolved from an 380 archaeal ancestor. Instead, the PVC-based linear scenario addresses most of the 381 inconsistencies of current 2D scenarios, and properly integrates most recent discoveries, 382 providing a coherent path of evolution of the three domains of life.

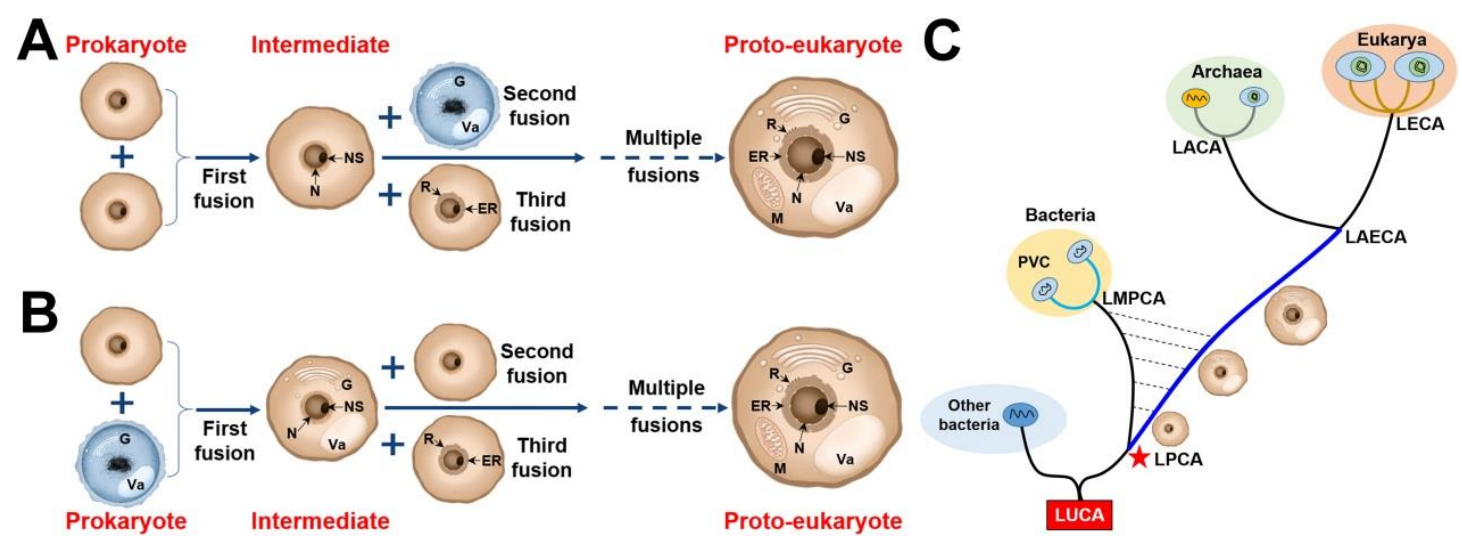

Fig. 6. Cell fusion drives PVC-based eukaryogenesis. (A) A proposed model of inter-species fusion of PVC bacteria launching the stepwise eukaryogenesis. ER, endoplasmic reticula; N, nucleus; NS, nucleolus; G, Golgi apparatus; Va, vacuole; M, mitochondria; R, ribosome. (B) A proposed model of cross-species fusion of PVC bacteria launching the stepwise eukaryogenesis. (C) PVC-based 1D tree of life. LUCA, the last universal common ancestor; LPCA, the last PVC common ancestor; LMPCA, the last modern PVC common ancestor; LAECA, the last archaeal and eukaryotic common ancestor; LACA, the last archaeal common ancestor; LECA, the last eukaryotic 


\section{Methods}

392 Isolation and cultivation of deep-sea PVC bacteria. The PVC bacterial strains 393 (including TYQ1, ZRK32, ZRK36 and zth2) were isolated from deep-sea deposits 394 obtained from a typical cold seep in the South China Sea by $R V K E X U E^{39,40}$. Strain 395 TYQ1 was obtained from an inoculum enriched in the aerobic MF7 medium 396 (containing $2.38 \mathrm{~g} / \mathrm{L}$ HEPES as a buffer, $5 \mathrm{~mL} / \mathrm{L}$ vitamin solution, $1 \mathrm{~mL} / \mathrm{L}$ trace 397 element solution, $0.25 \mathrm{~g} / \mathrm{L}$ glucose, $0.25 \mathrm{~g} / \mathrm{L}$ peptone, $0.25 \mathrm{~g} / \mathrm{L}$ yeast extract, $1 \mathrm{~g} / \mathrm{L}$ $398 \mathrm{~N}$-acetylglucosamine, $200 \mathrm{mg} / \mathrm{L}$ ampicillin, $100 \mathrm{mg} / \mathrm{L}$ carbenicilli, $200 \mathrm{mg} / \mathrm{L}$ 399 streptomycin, $20 \mathrm{mg} / \mathrm{L}$ amphotericin in $1 \mathrm{~L}$ filtered seawater, $\mathrm{pH}=7.0$ ). The enrichment was incubated at $28{ }^{\circ} \mathrm{C}$ for one week. Thereafter, enrichments were plated onto the 401 MF7 medium supplemented with $15 \mathrm{~g} / \mathrm{L}$ agar. Then small colorless colonies were 402 observed after one week and the strain TYQ1 was purified by continuous re-streaking 403 on MF7 solid plates until it was considered to be pure. Strains ZRK32, ZRK36 and zth2 404 were obtained from enrichments incubated in the anaerobic basal medium (containing $4051.0 \mathrm{~g} / \mathrm{L}$ yeast extract, $1.0 \mathrm{~g} / \mathrm{L}$ peptone, $1.0 \mathrm{~g} / \mathrm{L} \mathrm{NH}{ }_{4} \mathrm{Cl}, 1.0 \mathrm{~g} / \mathrm{L} \mathrm{NaHCO}_{3}, 1.0 \mathrm{~g} / \mathrm{L}$ $406 \mathrm{CH}_{3} \mathrm{COONa}, 0.5 \mathrm{~g} / \mathrm{L} \mathrm{KH}_{2} \mathrm{PO}_{4}, 0.2 \mathrm{~g} / \mathrm{L} \mathrm{MgSO}{ }_{4} \cdot 7 \mathrm{H}_{2} \mathrm{O}, 0.7 \mathrm{~g} / \mathrm{L}$ cysteine hydrochloride, $407500 \mu \mathrm{L} / \mathrm{L} 0.1 \%(\mathrm{w} / \mathrm{v})$ resazurin in filtered seawater, $\mathrm{pH}=7.0)$ supplemented with 50 $408 \mathrm{mg} / \mathrm{L}$ rifampicin or $1 \mathrm{~g} / \mathrm{L}$ laminarin at $28{ }^{\circ} \mathrm{C}$ for one month. Then $50 \mu \mathrm{L}$ enrichment was 409 spread on Hungate tubes covered by the basal medium supplemented with $15 \mathrm{~g} / \mathrm{L}$ agar

410 and incubated at $28{ }^{\circ} \mathrm{C}$ for 10 days. Individual colonies with distinct morphology were 411 picked using sterilized bamboo sticks and then cultured in the basal medium 412 supplemented with $50 \mathrm{mg} / \mathrm{L}$ rifampicin or $1 \mathrm{~g} / \mathrm{L}$ laminarin by repeated use of the 413 Hungate roll-tube methods for several rounds until they were considered to be pure ${ }^{41,42}$. 414 The purity of all strains (TYQ1, ZRK32, ZRK36 and zth2) were confirmed by 415 Transmission electron microscopy (TEM) and repeated partial sequencing of the $16 \mathrm{~S}$ 416 rRNA gene, then these pure bacterial strains were preserved at $-80{ }^{\circ} \mathrm{C}$ with $20 \%(\mathrm{v} / \mathrm{v})$ 417 glycerol.

418 Phylogenetic analysis. The full-length 16S rRNA gene sequences of four cultured 419 PVC bacterial strains (TYQ1, ZRK32, ZRK36 and zth2) were obtained from their 
genomes and deposited in the GenBank database, and other bacteria used for phylogenetic analysis were obtained from NCBI (www.ncbi.nlm.nih.gov). All the sequences were aligned by MAFFT version $7^{43}$ and manually corrected. The phylogenetic tree was constructed under the W-IQ-TREE web server (http://iqtree.cibiv.univie.ac.at) ${ }^{44}$ with $\mathrm{GTR}+\mathrm{F}+\mathrm{I}+\mathrm{G} 4$ model. And then this tree was edited by the online tool Interactive Tree of Life (iTOL v5) ${ }^{45}$.

TEM observation. To observe the morphological characteristics of PVC bacteria, the cell suspension was washed with the PBS buffer (containing $137 \mathrm{mM} \mathrm{NaCl}, 2.7 \mathrm{mM}$ $\mathrm{KCl}, 10 \mathrm{mM} \mathrm{Na}_{2} \mathrm{HPO}_{4}, 1.8 \mathrm{mM} \mathrm{KH}_{2} \mathrm{PO}_{4}, 1 \mathrm{~L}$ sterile water, $\mathrm{pH}$ 7.4) and centrifuged at $5,000 \times g$ for $10 \mathrm{~min}$, and taken by immersing copper grids coated with a carbon film for $30 \mathrm{~min}$, washed in the PBS buffer and air dried. For ultrastructure observation of PVC bacteria, ultrathin-section electron microscopy was performed as described previously $^{46}$. Briefly, four PVC strains were grown to the stationary phase in the rich medium and then centrifuged at $5,000 \times g$ for $10 \mathrm{~min}$. The samples were firstly preserved in $2.5 \%(\mathrm{v} / \mathrm{v})$ glutaraldehyde overnight at $4{ }^{\circ} \mathrm{C}$, washed three times with the PBS buffer and dehydrated in ethanol solutions of $30 \%, 50 \%, 70 \%, 90 \%$ and $100 \%$ for 10 min each time, and then the samples were embedded in a plastic resin. Ultrathin sections $(50 \sim 70 \mathrm{~nm})$ of cells were prepared with an ultramicrotome (Leica EM UC7, Wetzlar, Germany), stained with uranyl acetate and lead citrate. The ultrathin-sections of Bacillus thuringiensis A260, Pseudomonas stutzeri 273, Magnaporthe grisea and human liver cancer Huh 7.5 cell line were also prepared accordingly to the above procedures. All samples were observed using TEM (HT7700, Hitachi, Tokyo, Japan) with a JEOL JEM 12000 EX (equipped with a field emission gun) at $100 \mathrm{kV}^{39}$.

Genome sequencing and assembly. Whole-genome sequence determinations of four PVC strains (TYQ1, ZRK32, ZRK36 and zth2) were carried out with the Oxford Nanopore MinION (Oxford, UK) and Illumina MiSeq sequencing platform (San Diego,

46 California, USA). A complete description of the sequencing assembly and subsequent 447 analyses was performed as previously described ${ }^{39}$. 
448 Growth assay and transcriptomic analysis of strain ZRK32. To assess the effect of 449 organic nutrition on the growth of strain ZRK32, $15 \mathrm{~mL}$ freshly cells were inoculated in $450 \quad 1.5 \mathrm{~L}$ basal medium and rich medium at $28{ }^{\circ} \mathrm{C}$ for 7 days, respectively. Each condition 451 was performed three times. Bacterial growth status was monitored by measuring the $452 \mathrm{OD}_{600}$ value each day until the cell growth reached the stationary phase. For 453 transcriptomic analysis, cell suspensions of strain ZRK32 cultured in $1.5 \mathrm{~L}$ basal 454 medium or rich medium for 6 days were collected at $8,000 \times g$ for $20 \mathrm{~min}$. The 455 transcriptomic sequencing was performed by Novogene (Tianjin, China), and detailed 456 protocols including library preparation, clustering and sequencing and data analyses 457 were performed as previously described ${ }^{39}$.

458 Growth assay and transcriptomic analysis of strain ZRK36. Growth assays of 459 strain ZRK36 were performed at atmospheric pressure. Briefly, $15 \mathrm{~mL}$ fresh culture of 460 strain ZRK36 was inoculated in 2 L Hungate bottles containing $1.5 \mathrm{~L}$ basal medium, 461 and then anaerobically incubated at $28{ }^{\circ} \mathrm{C}$ for 7 days. And bacterial growth status was 462 monitored by measuring the $\mathrm{OD}_{600}$ value every $12 \mathrm{~h}$ until the cell growth reached the 463 stationary phase. For transcriptomic analysis, cell suspensions of strain ZRK36 464 cultured in $1.5 \mathrm{~L}$ basal medium for $2,4,5$ days were collected at $8,000 \times g$ for $20 \mathrm{~min}$, 465 respectively. Then, the transcriptomic sequencing and analysis were performed by 466 Novogene (Tianjin, China) as previously described ${ }^{39}$.

467 Data deposits and availability. The raw amplicon sequencing data have been 468 deposited to NCBI Short Read Archive (accession numbers: PRJNA768640). The 469 full-length 16S rRNA gene sequences of PVC superphylum strains (TYQ1, ZRK32, 470 ZRK36 and zth2) have been deposited at GenBank under the accession numbers: 471 OK160720, MW376756, MZ646149 and MW729759, respectively. The complete 472 genome sequences of PVC superphylum strains (TYQ1, ZRK32, ZRK36 and zth2) 473 have been deposited at GenBank under the accession numbers: CP083740, CP066225, $474 \mathrm{CP} 080649$ and $\mathrm{CP} 071032$, respectively. The raw sequencing reads from the 475 transcriptomics analysis of strain ZRK32 incubated in basal medium and rich medium 476 have been deposited to the NCBI Short Read Archive (accession numbers: 
477 PRJNA768630). The raw sequencing reads from the transcriptomics analysis of strain 478 ZRK36 at different stages of growth have been deposited to the NCBI Short Read 479 Archive (accession numbers: PRJNA768634 and PRJNA769601).

\section{Acknowledgements}

We thank Dr. Christina Lilliehook, from the professional editing group "Life Science Editors", for improving the scientific and writing quality of this manuscript. This work was funded by the Strategic Priority Research Program of the Chinese Academy of Sciences (Grant No. XDA22050301), the Major Research Plan of the National Natural Science Foundation (Grant No. 92051107), Key deployment projects of Center of Ocean Mega-Science of the Chinese Academy of Sciences (Grant No. COMS2020Q04), National Key R\&D Program of China (Grant No. 2018YFC0310800) and the Taishan Young Scholar Program of Shandong Province (tsqn20161051) for Chaomin Sun.

\section{Author contributions}

RZ and CS conceived and designed the study; RZ conducted most of the experiments; CW helped to do some experiments related to microscopic observation; TZ helped to isolate the Lentisphaerae bacterium; YT helped to isolate the Planctomycetes bacterium; RZ and CS lead the writing of the manuscript; all authors contributed to and reviewed the manuscript.

\section{Conflict of interest}

The authors declare no any competing interests.

\section{References}

1 Yamaguchi, M. et al. Prokaryote or eukaryote? A unique microorganism from the deep sea. J Electron Microsc 61, 423-431, doi:10.1093/jmicro/dfs062 (2012).

2 Gabaldon, T. Origin and early evolution of the eukaryotic cell. Annu Rev Microbiol 75, 631-647, doi:10.1146/annurev-micro-090817-062213 (2021).

3 Mikhailovsky, G. E. \& Gordon, R. LUCA to LECA, the Lucacene: A model for the gigayear delay from the first prokaryote to eukaryogenesis. Biosystems 205, doi: 
10.1016/j.biosystems.2021.104415 (2021).

4 Dacks, J. B. et al. The changing view of eukaryogenesis - fossils, cells, lineages and how they all come together. J Cell Sci 129, 3695-3703, doi:10.1242/jcs.178566 (2016).

5 de Roos, A. D. G. The origin of the eukaryotic cell based on conservation of existing interfaces. Artif Life 12, 513-523, doi:DOI 10.1162/artl.2006.12.4.513 (2006).

6 Baum, D. A. \& Baum, B. An inside-out origin for the eukaryotic cell. Bmc Biol 12, doi:10.1186/s12915-014-0076-2 (2014).

7 Embley, T. M. \& Williams, T. A. Steps on the road to eukaryotes. Nature 521, 169-170, doi:Doi 10.1038/Nature14522 (2015).

8 Poole, A. \& Penny, D. Engulfed by speculation. Nature 447, 913-913, doi:10.1038/447913a (2007).

9 Dacks, J. B. \& Field, M. C. Evolution of the eukaryotic membrane-trafficking system: origin, tempo and mode. J Cell Sci 120, 2977-2985, doi:10.1242/jcs.013250 (2007).

10 Devos, D. P. Reconciling Asgardarchaeota phylogenetic proximity to eukaryotes and Planctomycetes cellular features in the evolution of life. Mol Biol Evol 38, 3531-3542, doi:10.1093/molbev/msab186 (2021).

11 Rivas-Marin, E. \& Devos, D. P. The Paradigms They Are a-Changin': past, present and future of PVC bacteria research. Anton Leeuw Int $J G$ 111, 785-799, doi:10.1007/s10482-017-0962-z (2018).

12 Devos, D. P. \& Ward, N. L. Mind the PVCs. Environ Microbiol 16, 1217-1221, doi:10.1111/1462-2920.12349 (2014).

13 Orgel, L. E. The origin of life - a review of facts and speculations. Trends Biochem Sci 23, 491-495, doi:Doi 10.1016/S0968-0004(98)01300-0 (1998).

14 Sureka, R. \& Mishra, R. Identification of evolutionarily conserved nuclear matrix proteins and their prokaryotic origins. J Proteome Res 20, 518-530, doi:10.1021/acs.jproteome.0c00550 (2021).

15 Rothman, J. E. The machinery and principles of vesicle transport in the cell. Nat Med 8, 1059-1062, doi:10.1038/nm770 (2002).

16 Hay, J. C., Chao, D. S., Kuo, C. S. \& Scheller, R. H. Protein interactions regulating vesicle transport between the endoplasmic reticulum and Golgi apparatus in mammalian cells. Cell 89, 149-158, doi: 10.1016/S0092-8674(00)80191-9 (1997).

17 Zhang, C. H., Hicks, G. R. \& Raikhel, N. V. Plant vacuole morphology and vacuolar trafficking. Front Plant Sci 5, doi:10.3389/Fpls.2014.00476 (2014).

18 Shiratori, T., Suzuki, S., Kakizawa, Y. \& Ishida, K. Phagocytosis-like cell engulfment by a planctomycete bacterium. Nat Commun 10, doi:10.1038/S41467-019-13499-2 (2019).

19 Wiegand, S. et al. Cultivation and functional characterization of 79 planctomycetes uncovers their unique biology. Nat Microbiol 5, 126-140, doi:10.1038/s41564-019-0588-1 (2020). 
20 Sanderfoot, A. A., Assaad, F. F. \& Raikhel, N. V. The Arabidopsis genome. An abundance of soluble $\mathrm{N}$-ethylmaleimide-sensitive factor adaptor protein receptors. Plant Physiol 124, 1558-1569, doi: 10.1104/pp.124.4.1558 (2000).

21 Spang, A. et al. Complex archaea that bridge the gap between prokaryotes and eukaryotes. Nature 521, 173-179, doi:10.1038/nature14447 (2015).

22 Pollard, T. D. \& Cooper, J. A. Actin, a central player in cell shape and movement. Science 326, 1208-1212, doi:10.1126/science.1175862 (2009).

23 Wiegand, S., Jogler, M. \& Jogler, C. On the maverick Planctomycetes. Fems Microbiol Rev 42, 739-760, doi:10.1093/femsre/fuy029 (2018).

24 Pei, T. T. et al. Intramolecular chaperone-mediated secretion of an Rhs effector toxin by a type VI secretion system. Nat Commun 11, doi:10.1038/S41467-020-15774-Z (2020).

25 Rohs, P. D. A. \& Bernhardt, T. G. Growth and division of the peptidoglycan matrix. Annu Rev Microbiol 75, 315-336, doi:10.1146/annurev-micro-020518-120056 (2021).

26 Hendrickson, H. L. \& Poole, A. M. Manifold routes to a nucleus. Front Microbiol 9 , doi:10.3389/Fmicb.2018.02604 (2018).

27 Lusk, C. P. \& King, M. C. The nucleus: keeping it together by keeping it apart. Curr Opin Cell Biol 44, 44-50, doi:10.1016/j.ceb.2017.02.001 (2017).

28 Lafontaine, D. L. J., Riback, J. A., Bascetin, R. \& Brangwynne, C. P. The nucleolus as a multiphase liquid condensate. Nat Rev Mol Cell Bio 22, 165-182, doi:10.1038/s41580-020-0272-6 (2021).

29 Dey, G. et al. Closed mitosis requires local disassembly of the nuclear envelope. Nature 585, 119-123, doi:10.1038/s41586-020-2648-3 (2020).

30 Park, J. H., Jensen, B. C., Kifer, C. T. \& Parsons, M. A novel nucleolar G-protein conserved in eukaryotes. J Cell Sci 114, 173-185 (2001).

31 Klingauf-Nerurkar, P. et al. The GTPase Nog1 co-ordinates the assembly, maturation and quality control of distant ribosomal functional centers. Elife 9, doi:10.7554/eLife.52474 (2020).

32 Nagpal, H. et al. Dynamic changes in CCAN organization through CENP-C during cell-cycle progression. Mol Biol Cell 26, 3768-3776, doi:10.1091/mbc.E15-07-0531 (2015).

$33 \mathrm{Wu}, \mathrm{W}$. et al. CENPH inhibits rapamycin sensitivity by regulating GOLPH3-dependent mTOR signaling pathway in colorectal cancer. J Cancer 8, 2163-2172, doi:10.7150/jca.19940 (2017).

34 Santarella-Mellwig, R., Pruggnaller, S., Roos, N., Mattaj, I. W. \& Devos, D. P. Three-dimensional reconstruction of bacteria with a complex endomembrane system. Plos Biol 11, doi:10.1371/journal.pbio.1001565 (2013).

35 Sudhof, T. C. \& Rothman, J. E. Membrane fusion: grappling with SNARE and SM proteins. Science 323, 474-477, doi:10.1126/science.1161748 (2009).

36 Sauvola, C. W. \& Littleton, J. T. SNARE regulatory proteins in synaptic vesicle fusion and recycling. Front Mol Neurosci 14, doi:10.3389/Fnmol.2021.733138 (2021). 
37 Zaremba-Niedzwiedzka, K. et al. Asgard archaea illuminate the origin of eukaryotic cellular complexity. Nature 541, 353-358, doi:10.1038/nature21031 (2017).

38 Field, M. C. \& Rout, M. P. Pore timing: the evolutionary origins of the nucleus and nuclear pore complex. F1000Research 8, doi:10.12688/f1000research.16402.1 (2019).

39 Zheng, R. K. et al. Characterization of the first cultured free-living representative of Candidatus Izemoplasma uncovers its unique biology. ISME J 9, 2676-2691, doi:10.1038/s41396-021-00961-7 (2021).

40 Zhang, J. et al. A novel bacterial thiosulfate oxidation pathway provides a new clue about the formation of zero-valent sulfur in deep sea. ISME $J \mathbf{1 4}, 2261-2274$, doi:10.1038/s41396-020-0684-5 (2020).

41 Zheng, R. K., Cai, R., Liu, R., Liu, G. \& Sun, C. Maribellus comscasis sp. nov., a novel deep-sea Bacteroidetes bacterium, possessing a prominent capability of degrading cellulose. Environ Microbiol 23, 4561-4575, doi:10.1111/1462-2920.15650 (2021).

42 Zheng, R. K. \& Sun, C. Sphingosinithalassobacter tenebrarum sp. nov., isolated from a deep-sea cold seep. Int J Syst Evol Microbiol 70, 5561-5566, doi:10.1099/ijsem.0.004448 (2020).

43 Katoh, K., Rozewicki, J. \& Yamada, K. D. MAFFT online service: multiple sequence alignment, interactive sequence choice and visualization. Brief Bioinform. 20, 1160-1166, doi:10.1093/bib/bbx108 (2019).

44 Trifinopoulos, J., Nguyen, L. T., von Haeseler, A. \& Minh, B. Q. W-IQ-TREE: a fast online phylogenetic tool for maximum likelihood analysis. Nucleic Acids Res 44, W232-W235, doi:10.1093/nar/gkw256 (2016).

45 Letunic, I. \& Bork, P. Interactive Tree Of Life (iTOL) v5: an online tool for phylogenetic tree display and annotation. Nucleic Acids Res 49, W293-w296, doi:10.1093/nar/gkab301 (2021).

46 Graham, L. \& Orenstein, J. M. Processing tissue and cells for transmission electron microscopy in diagnostic pathology and research. Nat Protoc 2, 2439-2450, doi:10.1038/nprot.2007.304 (2007). 


\section{Expanded Data}

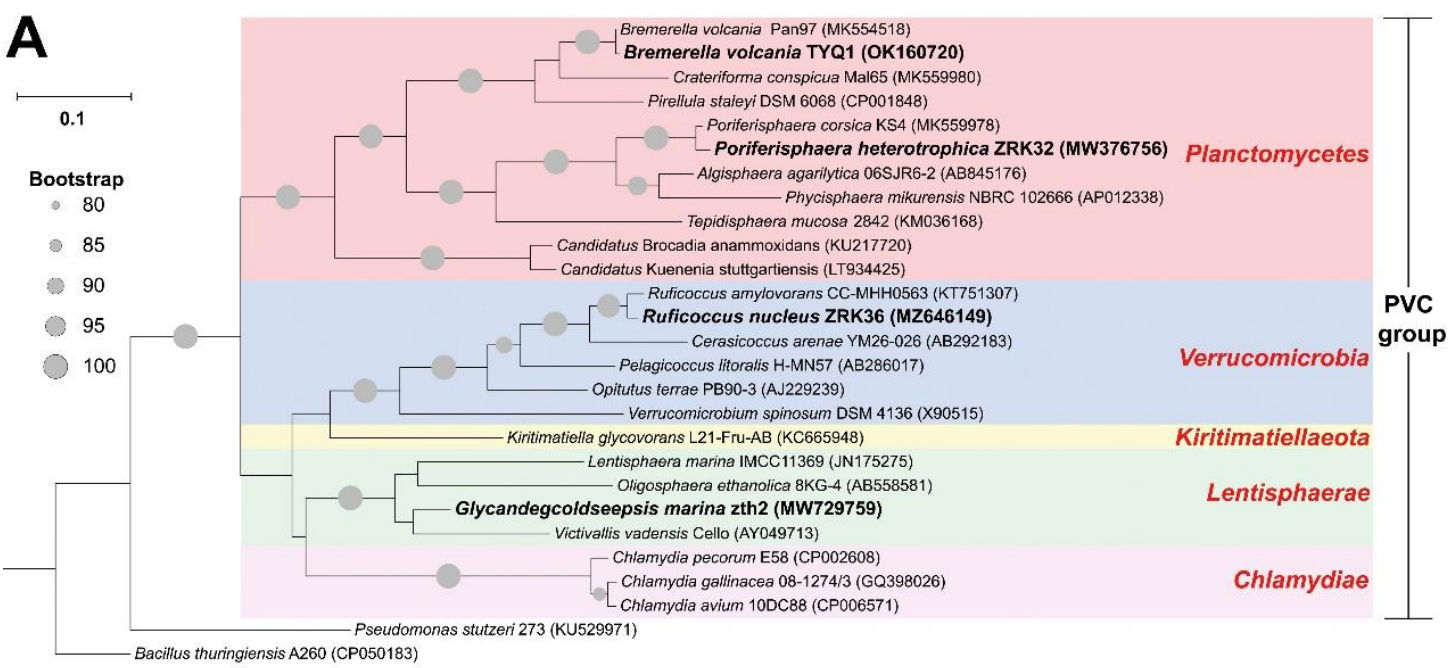

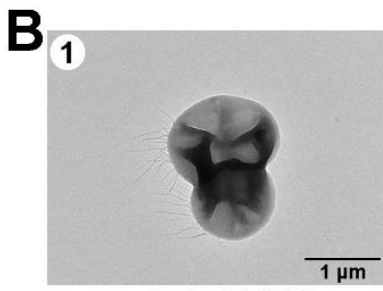

strain TYQ1
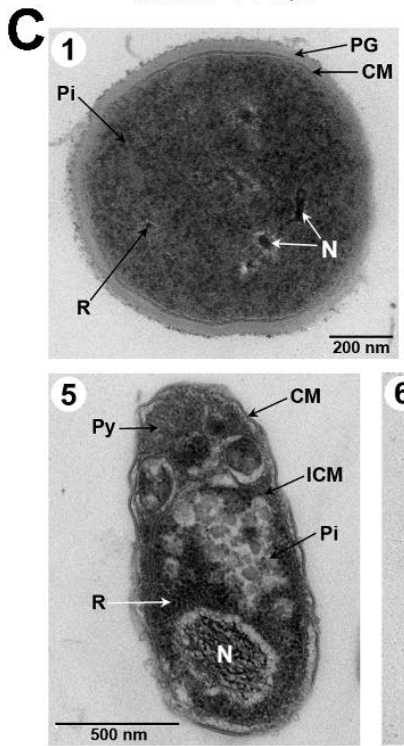

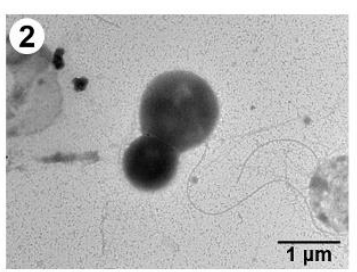

strain ZRK32
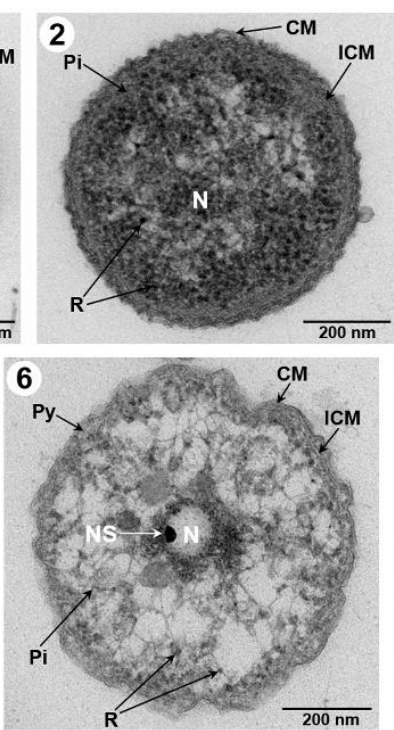

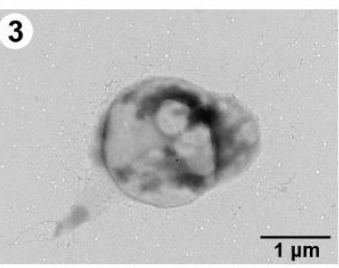

strain ZRK36
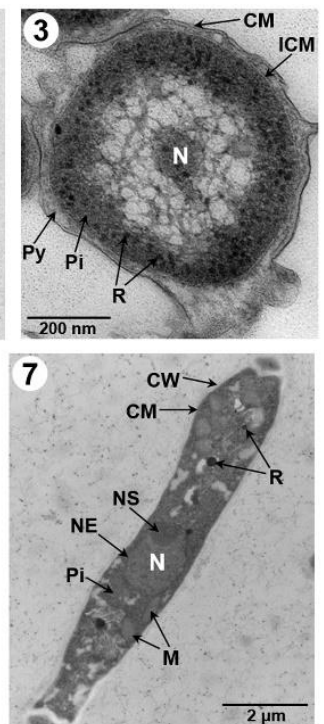

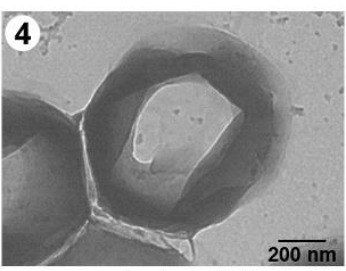

strain zth2
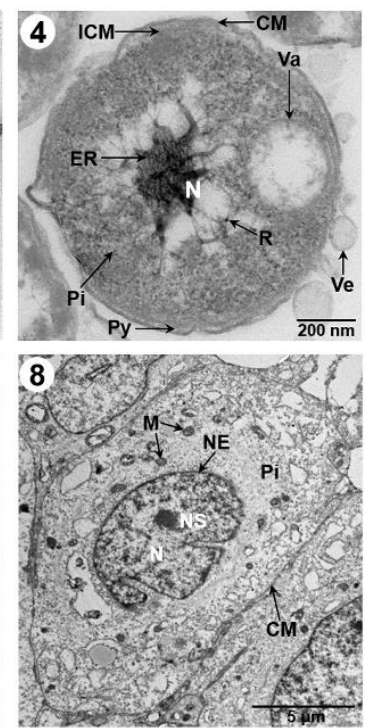

629 Expanded Data Fig. S1. Phylogeny and morphology of deep-sea novel members

630 belonging to the PVC superphylum. (A) 16S rRNA-based maximum-likelihood

631 phylogenetic tree shows the positions of novel deep-sea Lentisphaerae, Planctomycetes

632 and Verrucomicrobia strains are clustered within the PVC group. Bootstrap values (\%)>

63380 are indicated at the base of each node with the gray dots (expressed as percentages of

6341,000 replications). The access number of each 16S rRNA is indicated after the strain's 
635 name. The sequences of Pseudomonas stutzeri 273 and Bacillus thuringiensis A260 are

636 used as outgroup. Bar, 0.1 substitutions per nucleotide position. (B) TEM observation 637 of morphology of deep-sea Planctomycetes strains TYQ1 (panel 1) and ZRK32 (panel

638 2), Verrucomicrobia strain ZRK36 (panel 3) and Lentisphaerae strain zth2 (panel 4).

639 (C) Ultrathin TEM sections of Bacillus thuringiensis A260 (panel 1), Pseudomonas 640 stutzeri 273 (panel 2), Lentisphaerae strain zth2 (panel 3), Planctomycetes strains 641 ZRK32 (panel 4) and TYQ1 (panel 5), Verrucomicrobia strain ZRK36 (panel 6), 642 Magnaporthe grisea (panel 7) and human liver cancer Huh 7.5 cell line (panel 8). PG, 643 peptidoglycan; CM, cytoplasmic membrane; $\mathrm{Pi}$, pirellulosome; R, ribosome; N, 644 nucleoid or nucleus; ICM, intracytoplasmic membrane; Py, paryphoplasm; Va, vacuole 645 like-organelles; NS, nucleolus; NE, nucleus envelope; M, mitochondria.

646

647

648

649

650

651

652

653

654

655

656

657

658 

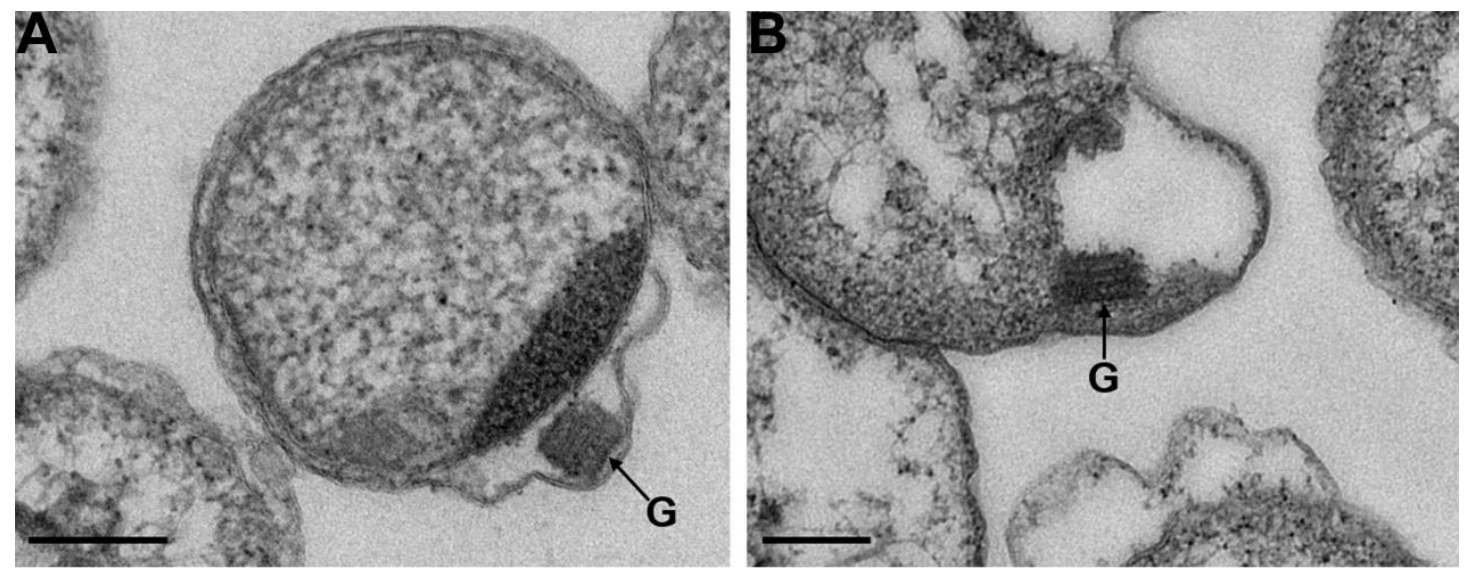

660 Expanded Data Fig. S2. Ultrathin TEM sections showing Golgi apparatus-like

661 organelle (indicated with G) observed in the cells of Planctomycetes strain ZRK32

662 (A-B). Bars: $200 \mathrm{~nm}$.

663

664

665

666

667

668

669

670

671

672

673

674

675

676

677

678

679

680

681 

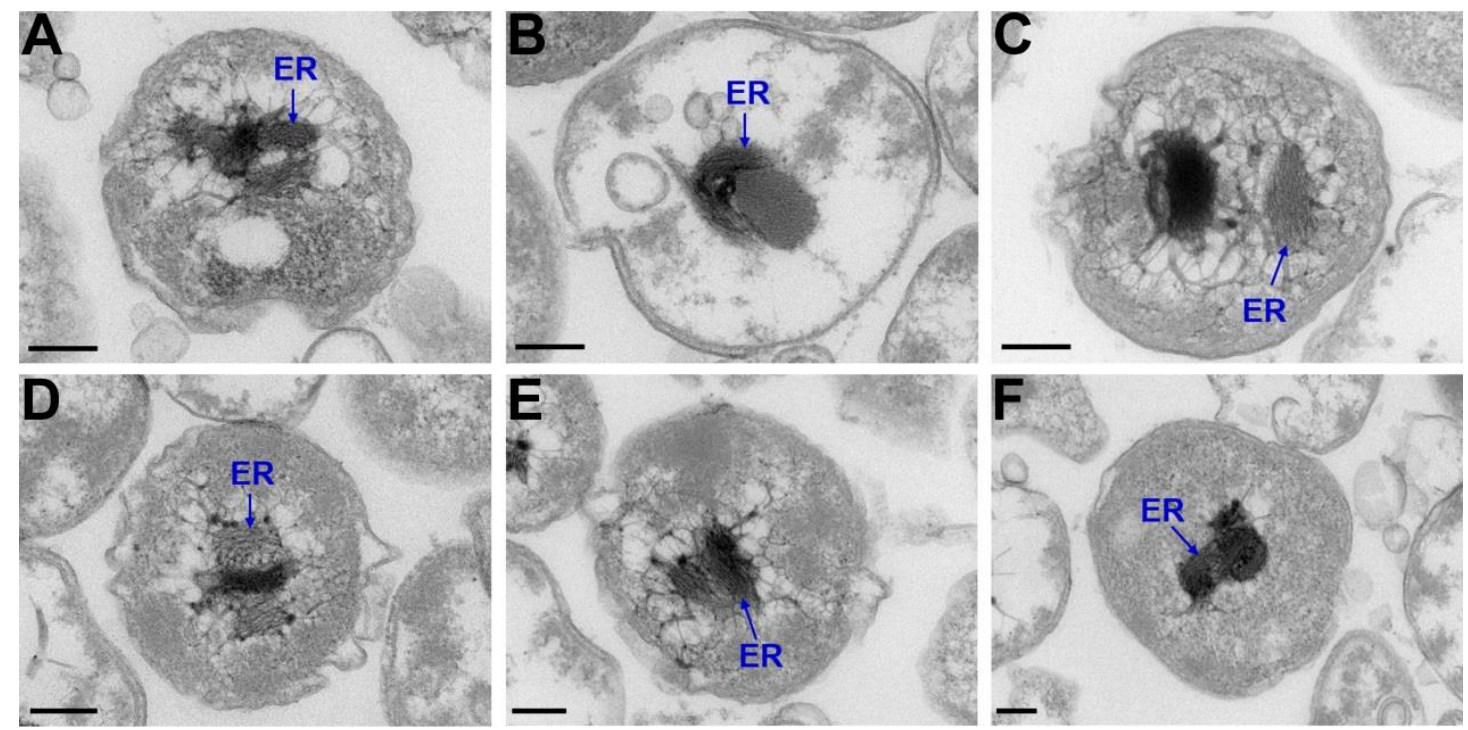

683 Expanded Data Fig. S3. Ultrathin TEM sections showing endoplasmic

684 reticula-like organelle (indicated with ER) observed in the cells of Planctomycetes

685 strain ZRK32 (A-F). Bars: $200 \mathrm{~nm}$.

686

687

688

689

690

691

692

693

694

695

696

697

698

699

700

701

702 

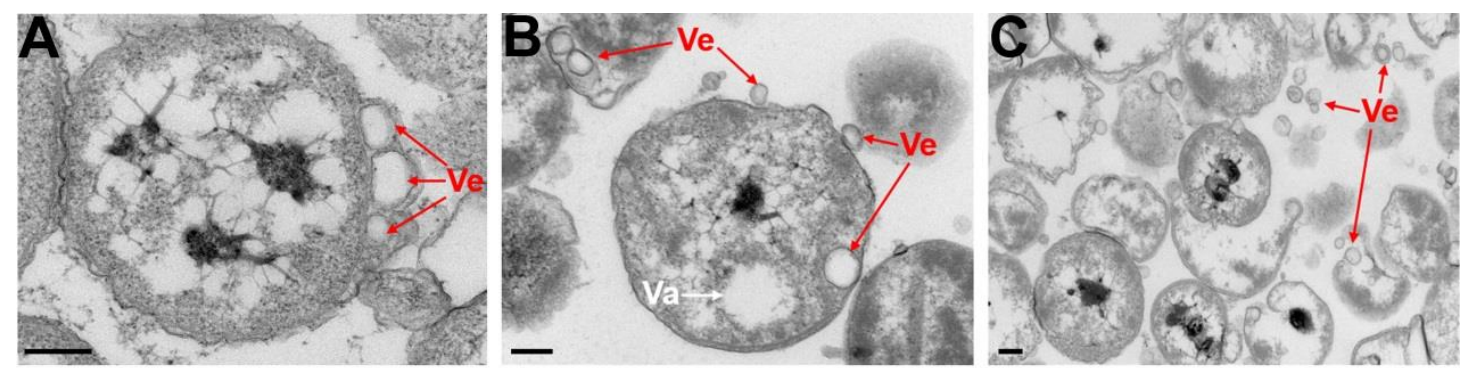
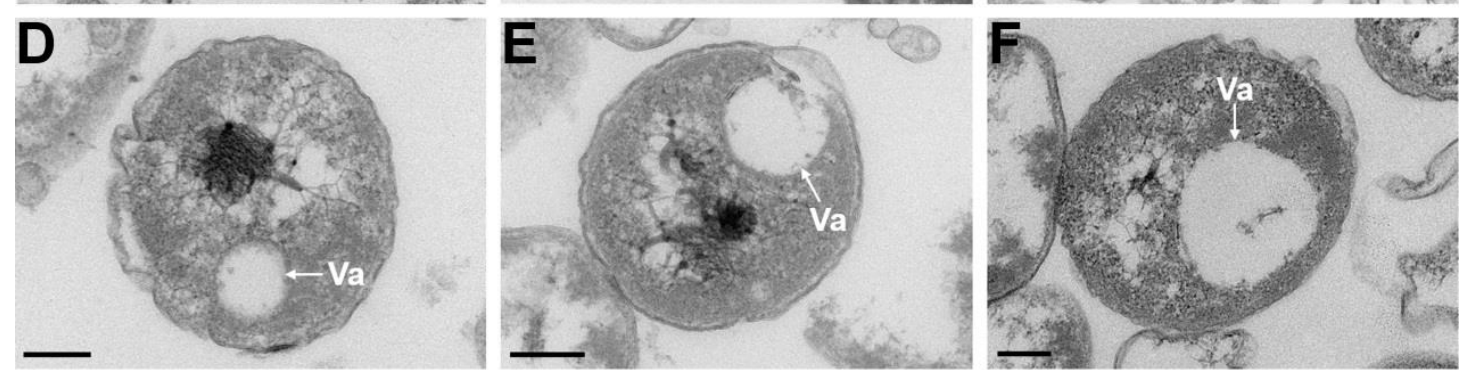

Expanded Data Fig. S4. Ultrathin TEM sections showing vesicle like- (indicated with Ve in panels A-C), vacuole like- (indicated with Va in panels D-F) organelles 

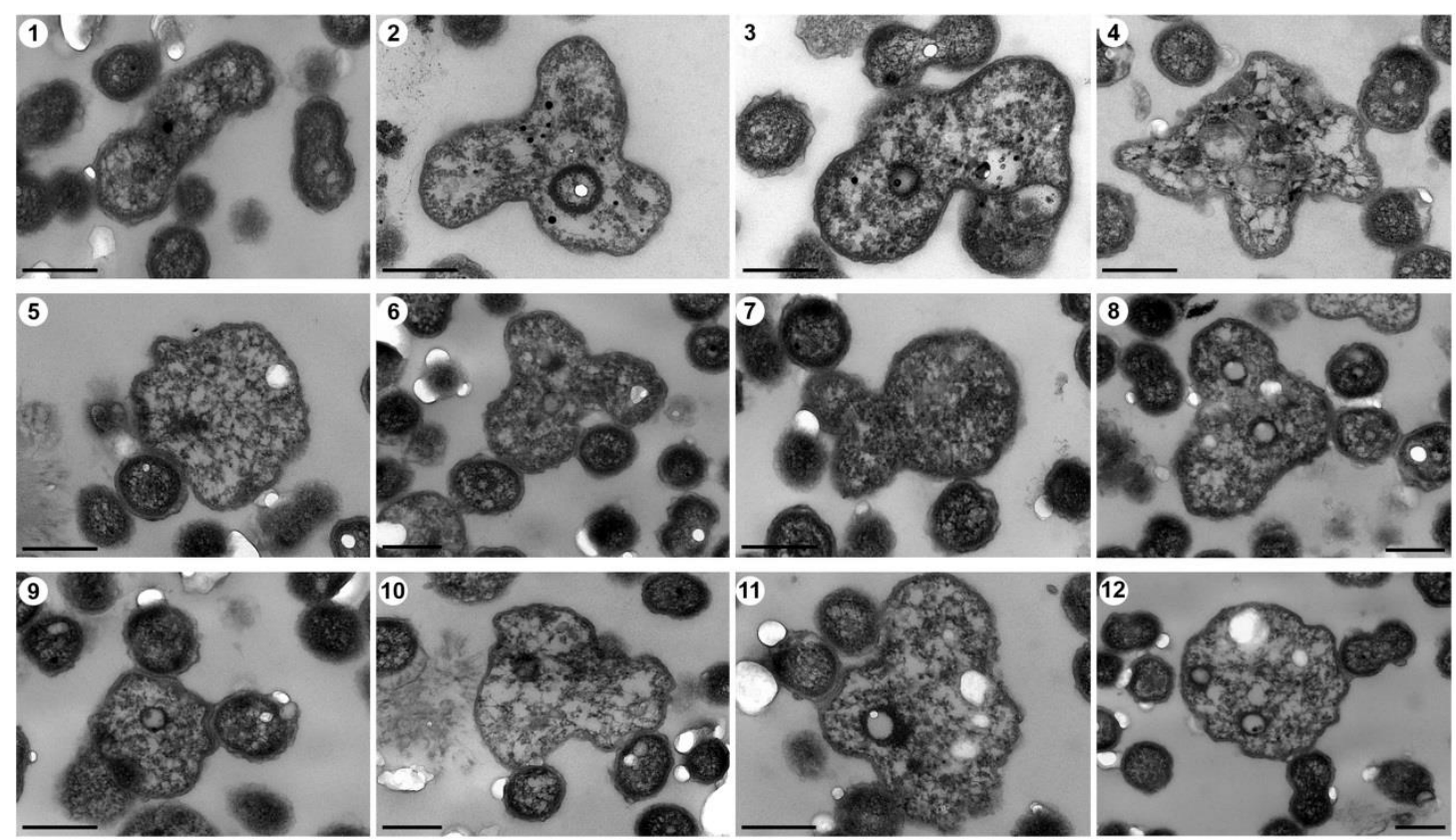

Expanded Data Fig. S5. Ultrathin TEM sections showing the phagocytosis process conducted by Verrucomicrobia strain ZRK36. 